\title{
Covalently deposited dyes: a new chromogen paradigm that facilitates analysis of multiple biomarkers in situ
}

\author{
William A Day, Mark R Lefever, Robert L Ochs, Anne Pedata, Lauren J Behman, Julia Ashworth-Sharpe, \\ Donald D Johnson, Eric J May, James G Grille, Esteban A Roberts, Jerry W Kosmeder and Larry E Morrison
}

Multiplexed analysis of multiple biomarkers in a tissue sample requires use of reporter dyes with specific spectral properties that enable discrimination of signals. Conventional chromogens with broad absorbance spectra, widely used in immunohistochemistry $(\mathrm{IHC})$, offer limited utility for multiplexed detection. Many dyes with narrow absorbance spectra, eg rhodamines, fluoresceins, and cyanines, potentially useful for multiplexed detection are well-characterized; however, generation of a chromogenic reagent useful for $\mathrm{IHC}$ analysis has not been demonstrated. Studies reported herein demonstrate utility of tyramine-chemistry for synthesis of a wide variety of new chromogenic dye conjugates useful for multiplexed in situ analysis using conventional light microscopes. The dyes, useful individually or in blends to generate new colors, provide signal sensitivity and dynamic range similar to conventional DAB chromogen, while enabling analysis of co-localized biomarkers. It is anticipated that this new paradigm will enable generation of a wide variety of new chromogens, useful for both research and clinical biomarker analysis that will benefit clinicians and patients.

Laboratory Investigation (2017) 97, 104-113; doi:10.1038/labinvest.2016.115; published online 21 November 2016

Modern histopathology is under increasing pressure to conserve tissue from biopsies while maximizing biomarker information. As a result there is a greater demand for multiplexing technologies that enable analysis of multiple biomarkers on a single tissue specimen. Indeed, increasing the number of biomarkers interrogated simultaneously has often improved the quality of diagnosis, providing greater association with clinical outcome (examples: esophageal, melanoma, and lung cancers), while also reducing consumption of limited clinical material. ${ }^{1-3}$ Historically, clinical evaluation of proteins and nucleic acids in tissue has relied upon in situ immunoenzymatic detection methods utilizing peroxidase and alkaline phosphatase to catalyze conversion of several well-known chromogenic stains, including 3,3'-diaminobenzidine (DAB), Fast Red, and Fast Blue, into insoluble products. ${ }^{4,5}$ These chromogens have broad absorbance spectra, which produce dark staining patterns that are easily distinguished against the light background of brightfield microscopy. In addition, DAB signals provide sufficient biomarker morphology and signal dynamic range to enable a number of predictive and prognostic immunohistochemical tests. Accordingly, DAB is the 'gold standard' chromogen used in the vast majority of advanced staining analyses executed and evaluated by pathologists worldwide. Unfortunately, while enabling high contrast, the broad absorbance spectra of $\mathrm{DAB}$ and other conventional chromogens limit the number of chromogens that can be visually distinguished from one another when applied to the same specimen. ${ }^{6}$ This limitation is particularly evident in the analysis of co-localized biomarkers that occupy the same cellular compartment. In addition, DAB precipitation can produce a diffuse deposition pattern that, although currently sufficient for a number of prognostic and predictive tests, may not reflect biomarker biology and may compromise analysis of biologically relevant information. Collectively, these observations suggest that $\mathrm{DAB}$ and conventional chromogens, though useful for detection of individual biomarkers, are limited in their utility for multiplexed detection of multiple biomarkers in tissue samples. Indeed, the need to conserve specimen, accurately identify expression of co-localized biomarkers, and generate signals that accurately reflect multiple biomarker biology creates a necessity for new chromogens.

One way to achieve better color discrimination in multiplexed chromogenic assays would be to utilize dyes that strongly absorb relatively narrow bands of light so that each dye removes only a small fraction of the visible spectrum.

Tissue Research and Early Development, Ventana Medical Systems, Inc., Tucson, AZ, USA

Correspondence: Dr LE Morrison, PhD, Tissue Research and Early Development, Ventana Medical Systems, Inc., 1910 E. Innovation Park Drive, Tucson, AZ 85755 , USA. E-mail: larry.morrison@roche.com

Received 19 May 2016; revised 6 September 2016; accepted 3 October 2016 
Use of dyes with these properties in a multiplexed format would permit fitting more dyes within the visible spectrum and facilitate evaluation of co-localized biomarkers since overlapping dyes would collectively absorb more of the visible spectrum than either dye individually and still transmit appreciable spectrally distinct portions of the spectrum. Many common dyes possess these desirable spectral properties. However, modifying dyes to possess the necessary characteristics for in situ staining, ie, water-soluble substrate forms enzymatically convertible to highly insoluble products, would be challenging and differ for each dye.

The demands on new chromogen development could be greatly reduced if solubility was no longer relied upon as the means to deposit the chromogen. Highly reactive products of enzymatic reactions offer a means to rapidly localize dyes to tissue, via covalent attachment in close proximity to the biomarker, thereby accurately reflecting biomarker distribution. Methods to covalently attach dye molecules to tissue specimens have been described. For example, peroxidasecatalyzed oxidation of cyanine dyes can produce reactive dye derivatives that covalently attach to histological specimens producing chromogenic staining patterns. ${ }^{7}$ However, this approach is dependent upon unique properties of cyanine dyes and is of limited or no use with other dye families. A more general means of generating highly reactive species is provided by tyramine substituents which, upon peroxidasecatalyzed oxidation, form highly reactive free radicals that covalently attach to electron-rich moieties, such as certain amino acids found in proteins adsorbed to surfaces in enzyme immunoassays and within cellular and tissue specimens in immunohistochemistry (IHC) and in situ hybridization (ISH) assays. $^{8-15}$ Consequently, any dye conjugated to the tyramine also becomes covalently bound to the tissue. Conjugation of tyramines via their primary amine to dyes with amine-reactive substituents is a straightforward procedure and has been successfully used with a variety of commercially available fluorophores for in situ deposition of immunofluorescence. ${ }^{12,14,16-19}$ However, tyramine chemistry has not been reported for chromogen deposition in IHC or ISH in which the chromogen dye becomes covalently attached to cellular material, or tissue.

Here we demonstrate for the first time that conjugation of tyramine to dyes can produce a number of spectrally distinct chromogens, and these chromogens can be effectively integrated into multiplexed brightfield in situ assays. The new chromogens are examined visually and spectrally and are found to provide DAB-like performance, providing the basis for both effective chromogen deposition and easily selectable spectral properties, leading to rapid development of new chromogens suitable for multiplexed chromogenic in situ assays of protein and nucleic acid targets. This general approach, using a common activable linker unit, may provide a new paradigm for rapid creation of new chromogens with properties well suited to multiplexed biomarker analysis.

\section{MATERIALS AND METHODS Synthesis of Tyramide Chromogens}

See Supplementary Methods for details regarding synthesis of each tyramide-dye conjugate. Tyramide-dye conjugates were solubilized in dimethyl sulfoxide (DMSO) and the resulting stock solutions stored at $4{ }^{\circ} \mathrm{C}$.

\section{Immunohistochemistry}

All chemicals (except DMSO), reagents, and instruments were manufactured by Ventana Medical Systems (Tucson, AZ). Automated non-multiplexed detection of single proteins (Bcl-2, Her2, HMB45, and Ki-67) in standard CC1-antigen retrieved slides was accomplished using un-modified native primary antibodies and OmniVIEW or UltraView detection reagents, and a VENTANA BenchMark XT instrument as directed by the manufacturer. Automated multiplexed detection of $\mathrm{CD} 3, \mathrm{CD} 8, \mathrm{CD} 68$, cytokeratin, and $\mathrm{Ki}-67$ proteins in standard CC1-antigen retrieved slides was accomplished using sequential application of unmodified native antibody (anti-Ki-67), UltraView HRP conjugated anti-species antibodies, and tyramide-dye conjugates, followed by application of cocktailed hapten-conjugated anti-target monoclonal antibodies (anti-CD3, anti-CD8, anti-CD68, and anti-cytokeratin), followed by sequential application of cognate HRP-conjugated anti-hapten monoclonal antibodies and tyramine-dye conjugates using a Benchmark XT instrument. Inactivation of HRP activity between sequential detection steps was accomplished using three successive applications of three drops of Discovery reagent PO Inhibitor incubated for 12 min each. Tyramide-dye conjugate stocks were diluted in buffer containing 10\% DMSO (to maintain solubility) and placed in an instrument dispenser. One drop $(100 \mu \mathrm{l})$ of dilute tyramide-dye was dispensed onto slides overlayed with reaction buffer containing one drop $(100 \mu \mathrm{l})$ of hydrogen peroxide solution. Tyramide-dye/HRP chromogen activation and covalent binding reactions were incubated for $40 \mathrm{~min}$ at $37^{\circ} \mathrm{C}$. IHC using the conventional chromogens Fast Red and Fast Blue were performed with the UltraView Universal Alkaline Phosphatase Red and ISH iVIEW Blue $_{\text {Plus Detection }}$ Kits per manufacturer's instructions.

\section{In Situ Hybridization}

KAPPA constant light-chain mRNA target was detected using a hapten-tyramide amplification, chromogenic system and a BenchMark XT instrument. Briefly, antisense DNP-labeled probe was hybridized to de-paraffinized, mild CC1-retrieved, protease treated tissue samples. Following stringency washes the haptens were sequentially detected using an anti-hapten monoclonal antibody conjugated to HRP which catalyzed deposition of a tyramide-hapten conjugate; the amplified hapten was then detected using a second anti-hapten antibody-HRP conjugate to catalyze chromogen deposition. The slides were then stained with hematoxylin to visualize the nuclei. Following staining, all tyramide-conjugate-stained 
slides were dehydrated using an ethanol gradient and coverslipped using a xylene-based mounting medium.

\section{Microscopy}

Stained specimens were viewed on a Zeiss Axio Imager.A1 configured for brightfield microscopy and equipped with a Zeiss AxioCam MRc5 color CCD camera for recording microscopic images.

\section{Spectroscopy}

Absorbance spectra of dye-tyramide conjugates in solution were recorded on an Agilent 8453 Spectrophotometer. Absorbance spectra of deposited chromogens were recorded on slide-mounted specimens placed on the stage of an Olympus BX-63 microscope under tungsten illumination. Transmitted light was measured between 350 and $800 \mathrm{~nm}$ in $\sim 0.5 \mathrm{~nm}$ increments using a Pryor Scientific (Rockland, MA) Lumaspec 800 power meter. The spectrum of light transmitted through a stained region of the slide was divided by the spectrum transmitted through on an unstained region to provide the transmission $(\mathrm{T})$ spectrum, which was converted to the chromogen absorbance (A) spectrum using the relationship $A=\log _{10}(1 / T)$. Absorbance values of individual punctate spots of stained mRNA molecules were recorded using an Applied Spectral Imaging (Carlsbad, CA) hyperspectral imaging camera mounted on a Zeiss Axio Imager.M2 microscope.

\section{RESULTS}

Synthesis and Characterization of Tyramide Chromogens Ideal properties for new chromogens include narrow absorption bands that occupy a minimal range of the visible spectrum to increase multiplexing capacity, and sufficiently high extinction coefficients $\left(>10000 \mathrm{M}^{-1} \mathrm{~cm}^{-1}\right)$ such that the narrow absorption dyes absorb sufficient light to generate a visible signal. A number of commercially available dyes were identified possessing these properties and coupled to tyramine to form dye-tyramides. Published wavelengths of peak absorbance, full width of the absorbance bands at half the peak absorbance (full width at half maximum, FWHM), and molar extinction coefficients for seven of the dyes prior to conjugation are listed in Table 1, columns 2, 3, and 4 (manufacturer or literature values). Solubility of aqueous solutions of dye-tyramides was improved by placing a hydrophilic PEG8 linker between the dye and tyramide portions of the conjugate. DMSO (10\%) was added to stock solutions to assure solubility. Fourteen dye-conjugates were generated that provided acceptable solubility at the concentrations required for peroxidase-catalyzed chromogen deposition and for concentrated stock solutions used to fill the autostainer dispensers (see below). Spectral properties of the resulting dye-tyramides in solution are listed in Table 1 (columns 7, 8) for the seven dye-tyramides selected for further work.

\section{In Situ Deposition of Tyramide Chromogens}

Utility of each dye-tyramide conjugate as a chromogenic stain for detecting protein in formaldehyde-fixed paraffinembedded tissue samples was determined using commercial immunohistochemical (IHC) reagents, an automated tissue stainer, and Calu-3 and MCF-7 xenograft models expressing high (3+) and low (0+) amounts of erbb2 receptor tyrosine kinase 2 (ERBB2; commonly referred to as Her2) protein targets, respectively. A range of concentrations was employed to empirically determine each potential chromogen's utility for sensitive and specific signal generation compared with signals generated using the 'gold standard' DAB chromogen. Slides stained using the dye-tyramide conjugates were processed using standard dehydration steps identical to those used to process DAB stained slides. Effective dye concentrations for peroxidase-catalyzed deposition ranged from $100 \mu \mathrm{M}$ to $600 \mu \mathrm{M}$, depending upon the particular dyetyramide (Table 1, column 5) and were automatically diluted from concentrated stock solutions in the autostainer dispensers, ranging between $500 \mu \mathrm{M}$ and $3 \mathrm{mM}$ (Table 1, column 6). Results of IHC staining using fourteen of the dyetyramide chromogens applied to high Her2-expressing (3+) xenografts are pictured in Figure 2a, showing staining patterns consistent with the DAB staining. Also consistent with DAB, no signals were observed in the low Her2-expressing (0+) MCF-7 xenograft tissue (data not shown) and, as observed in several images (Figure 2a), no signals were observed in murine fibroblasts, which do not express Her2 demonstrating each dye's specificity. Generally, signals generated using the dye-tyramides were consistent in both morphology and specificity to those generated using conventional chromogen (DAB). In addition, colors of the observed signals were consistent with each dye's spectral characteristics. Visual color of each deposited chromogen is listed in Table 1, column 11. As the spectral properties might be altered by deposition in tissue, absorbance spectra of dye-tyramides deposited by IHC were recorded and plotted in Figure 1a. Peak absorption wavelengths and FWHM of the deposited dye spectra are listed in columns 9 and 10 of Table 1. For comparison with conventional chromogens, spectra of several common chromogens deposited by IHC are plotted in Figure 1b, and absorption peaks and FWHM are also listed (Table 1, columns 9 and 10). In general, the peak absorbance wavelengths and bandwidths are similar between the published values for unconjugated dyes, the dye-tyramides in solution, and the corresponding dye-tyramides deposited onto tissue. The major differences in absorbance spectra were several modest shifts of the peak absorbance values and some broadening of the deposited dye absorbance bands. Even with some broadening, six of the seven deposited dyes in Table 1 had substantially narrower absorbance bands than the conventional dyes, and the remaining dye, 4-(dimethylamino)azobenzene- 4 '-sulfonate (dabsyl), had a bandwidth similar to the narrowest of the conventional dyes. In addition, 
Table 1 Spectral properties of dyes prior to and after conjugation to tyramine, and after covalent deposition via peroxidase-catalyzed oxidation of conjugate, and working concentrations

\begin{tabular}{|c|c|c|c|c|c|c|c|c|c|c|}
\hline \multirow[t]{2}{*}{ Dye } & \multicolumn{3}{|c|}{ Unconjugated dye spectral properties* } & \multirow{2}{*}{$\begin{array}{c}\text { Reaction } \\
\text { concentration } \\
(\mathrm{mM})\end{array}$} & \multirow{2}{*}{$\begin{array}{c}\text { Stock } \\
\text { concentration } \\
(\mathrm{mM})\end{array}$} & \multicolumn{2}{|c|}{$\begin{array}{l}\text { Conjgated dye spectral } \\
\text { properties in solution }\end{array}$} & \multicolumn{2}{|c|}{$\begin{array}{l}\text { Deposited dye-tyramide spectral } \\
\text { prioperties }\end{array}$} & \multirow{2}{*}{$\begin{array}{l}\text { Visual color of } \\
\text { deposited dye } \\
\text { conjugate }\end{array}$} \\
\hline & $\begin{array}{c}\text { Peak } \\
\text { Absorbance } \\
(\lambda, \mathrm{nm})\end{array}$ & $\begin{array}{c}\mathrm{FWHM}(\lambda, \\
\mathrm{nm})\end{array}$ & $\varepsilon\left(\mathrm{M}^{-1} \mathrm{~cm}^{-1}\right)$ & & & $\begin{array}{c}\text { Peak } \\
\text { Absorbance } \\
(\lambda, \mathrm{nm})\end{array}$ & $\begin{array}{c}\text { FWHM }(\lambda, \\
\mathrm{nm})\end{array}$ & $\begin{array}{c}\text { Peak } \\
\text { Absorbance } \\
(\lambda, \mathrm{nm})\end{array}$ & FWHM $(\lambda, \mathrm{nm})$ & \\
\hline \multicolumn{11}{|c|}{ Covalently deposited chromogens } \\
\hline Dabsyl & $469-476^{a}$ & $110^{\mathrm{b}}$ & $\begin{array}{l}10100- \\
44500^{a}\end{array}$ & 0.6 & 3 & 478 & 113 & 455 & 118 & Yellow \\
\hline Rhod 110 & $497^{c}$ & $37^{c}$ & $76000^{c}$ & 0.2 & 1 & 501 & 37 & 509 & 66 & Yellow-brown/red \\
\hline Rhod $6 \mathrm{G}$ & $525^{c}$ & $51^{c}$ & $100000^{c}$ & 0.1 & 0.5 & 528 & 41 & 529 & 54 & magenta \\
\hline TAMRA & $552^{e}$ & $37^{\mathrm{e}}$ & $95000^{\mathrm{e}}$ & 0.2 & 1 & 554 & 38 & 552 & 56 & Magenta \\
\hline SRhod B & $568^{f}$ & $52^{9}$ & $88000^{f}$ & 0.2 & 1 & 570 & 34 & 568 & 43 & Magenta \\
\hline SRhod 101 & $595^{e}$ & $34^{e}$ & $100000^{e}$ & 0.1 & 0.5 & 591 & 49 & 579 & 74 & Purple-blue $^{d}$ \\
\hline Cy5 & $649^{e}$ & $39^{e}$ & $250000^{e}$ & 0.2 & 1 & 648 & 36 & 669 & 68 & Cyan-blue $^{d}$ \\
\hline \multicolumn{11}{|c|}{ Conventional precipitating chromogens: } \\
\hline$D A B$ & & & & & & & & 464 & $>300$ & Brown \\
\hline Fast Red & & & & & & & & 525 & 109 & Red \\
\hline Fast Blue & & & & & & & & 607 & 184 & Blue \\
\hline
\end{tabular}

Abbreviations: DAB, 3,3'-diaminobenzidine; DABSYL, 4-(4-dimethylaminophenylazo)benzenesulfonyl; FWHM, full width at half maximum; Rhod, rhodamine; Srhod, sulforhodamine; TAMRA, carboxytetramethylrhodamine; $\varepsilon$, extinction coefficient for absorbance; $\lambda$, wavelength.

Spectral properties of several convention dyes are also included for comparison.

a Range for sulfonamides of DABSYL with various amino acids in $0.1 \mathrm{M} \mathrm{NaHCO}_{3}{ }^{24}$

${ }^{b}$ Sulfonamide of DABSYL with tyramine in dioxane. ${ }^{25}$

'Aqueous solution, $\mathrm{pH} 7$; ThermoFisher/Invitrogen/Molecular Probes product literature including spectra data files.

${ }^{\mathrm{d} C o l o r ~ d e p e n d e n t ~ u p o n ~ a m o u n t ~ o f ~ d e p o s i t e d ~ d y e . ~}$

${ }^{\mathrm{e}}$ Attached to antibody; ThermoFisher/Invitrogen/Molecular Probes product literature including spectra data files.

${ }^{f}$ Methanol; ThermoFisher/Invitrogen/Molecular Probes product literature including spectra data files.

${ }^{9}$ Aqueous solution. ${ }^{26}$ 

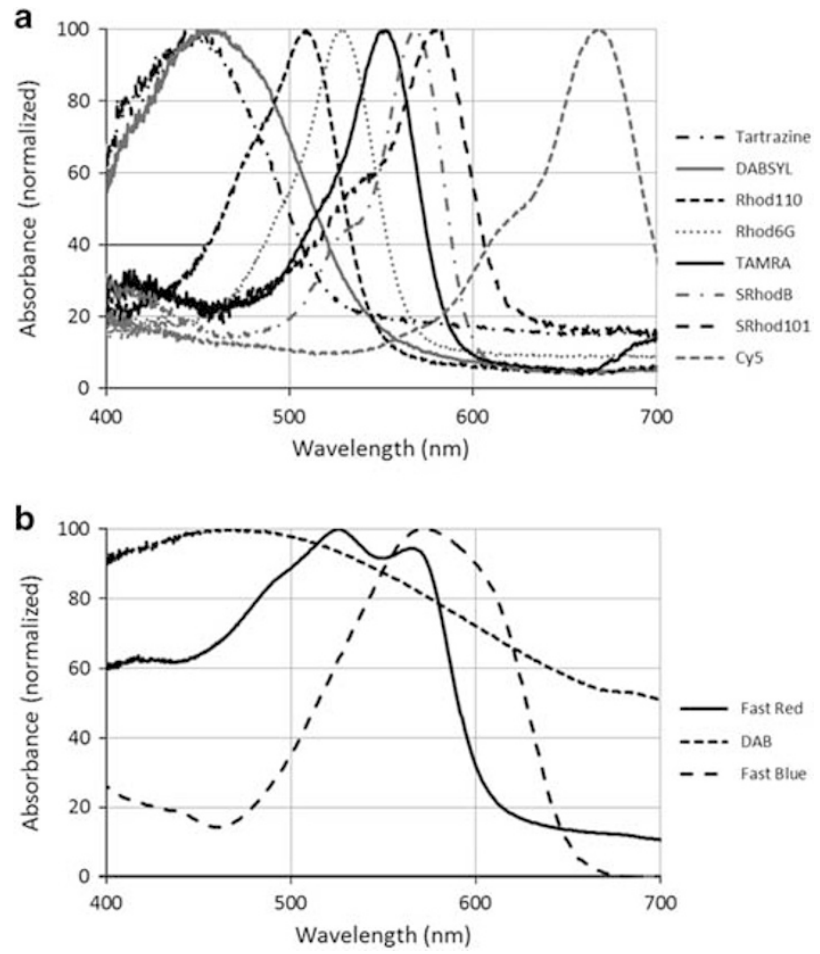

Figure 1 Absorbance spectra of dye-tyramide chromogens (a) and conventional chromogens (b) deposited on FFPE tissue specimens. Absorption spectra are normalized to a value of 100 at the wavelengths of maximum absorbance.

time-lapse studies of several dye-tyramide stock solutions demonstrated greater than 3-month stability in solution (data not shown).

In addition to IHC protein stains, the new chromogens were applied to in situ hybridization for detection of nucleic acid (RNA) as seen in Figure 2b. Each tyramide-dye conjugate generated a range of signals consistent with both KAPPA constant light-chain mRNA abundance in lymphoid follicale $\mathrm{B}$ cells and the punctate staining pattern typical of RNA ISH. ${ }^{20,21}$ Images of stained specimens were used to estimate the amount of dye covalently deposited employing an interferometer-based spectral imaging system to measure the dye absorbance of the carboxytetramethylrhodamine (TAMRA) tyramide chromogen deposited in small punctate spots of different staining intensities. Lightly staining single spots $(A \sim 0.1)$, covering approximately $0.4 \mu \mathrm{m}^{2}$, were determined to contain roughly 2 million TAMRA molecules and darkly staining spots $(A \sim 0.5)$ of similar size contained roughly 10 million TAMRA molecules (see Supplementary Information for details).

Close inspection of IHC staining revealed a range of signal characteristics with some dependence upon particular dyes. In particular, rhodamine-based dye signals appeared to have more definition (punctate signal) compared with nonrhodamine tyramide dyes or $\mathrm{DAB}$ chromogen. This property was further evaluated by IHC staining for HMB45 expressing granuoles in the melanocyte cytoplasm. Individual HMB45expressing granuoles were observed using the rhodaminebased dye TAMRA; in contrast, no individual granuoles were discernable using conventional DAB or Fast Red chromogens (Figure 3 and data not shown).

Immunohistochemical tests are routinely used to interrogate expression of clinically useful protein biomarkers in various cellular compartments (nuclear, cytoplasmic, and membrane) over a wide dynamic range. Accordingly, model nuclear (Ki-67) and cytoplasmic (cytokeratin) proteins, expressed over a wide dynamic range in tonsil tissues, were used to determine utility of three disparate types of dyetyramides: four rhodamine dyes (rhodamine 110, rhodamine 6G, TAMRA, and sulforhodamine 101), a cyanine dye (Cy-5), and a diaminobenzene dye (dabsyl) to detect dynamically expressed protein targets in various cellular compartments. Each dye generated a range of nuclear and cytoplasmic signals consistent with those observed using DAB chromogen (Figure $4 a-k)$, indicating similar dynamic range in each cellular compartment.

\section{Chromogen Mixes Generate New Colors}

Several of the new chromogens with relatively narrow absorbance spectra were tested for their ability to create unique colors when mixed together and co-deposited by IHC. Accordingly, Cy5- and dabsyl-tyramides were mixed in various ratios and used as substrates of HRP antibody conjugates in the Her2 3+ IHC model. Color images of the results are pictured in Figure 5a with cyan staining of Cy5-tyramide alone in the left panel, yellow staining of dabsyl-tyramide alone in the right panel, and the resulting green staining of co-deposited Cy-5-tyramide and dabsyltyramide in the center panel. Similarly, mixing of TAMRA and dabsyl-tyramides is pictured in Figure 5b, with TAMRAtyramide alone producing magenta stain (left panel), dabsyltyramide alone producing yellow stain (right panel), and co-deposition of the two chromogens producing red stain (center panel). Finally, Figure $5 \mathrm{c}$ features mixing of Cy5- and TAMRA-tyramides, with cyan staining of Cy5-tyramide alone (left panel), magenta staining of TAMRA-tyramide alone (right panel), and blue staining of the co-deposited Cy5- and TAMRA-tyramides (center panel).

Successful generation of new colors through co-deposition of mixed chromogens suggested a new ability to identify colocalized expression of protein biomarkers in a single cellular compartment. Evaluation of this utility was performed using two nuclear proteins (Bcl-2 and Ki-67) variably expressed in tonsil germinal center cells. As indicated in Figure 5d, individual expression of each protein in tonsil lymphocytes was observed as non-mixed signal resulting from the parental dye color, either yellow staining of Ki-67 by dabsyl-tyramide, or magenta staining of Bcl-6 by TAMRA-tyramide. In contrast, expression of both proteins within an individual lymphocyte nucleus was observed as a range of reddish staining, likely reflecting dynamic expression of each protein. These results 
demonstrate that co-expression of two biomarkers can be convincingly identified by the overlay of two narrow band absorbing chromogens.

\section{Multiplexed Protein Detection}

The main impetus for developing covalent dye deposition technology was to facilitate simultaneous detection of

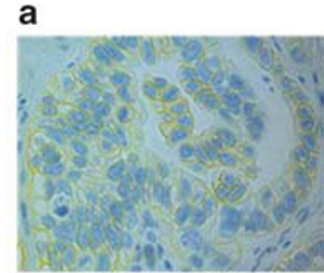

Tartrazine

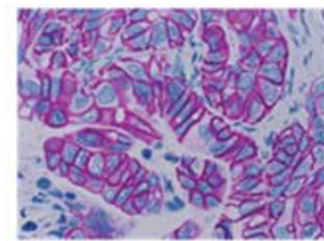

Tetramethylrhodamine

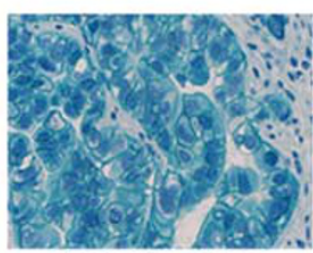

Victoria Blue

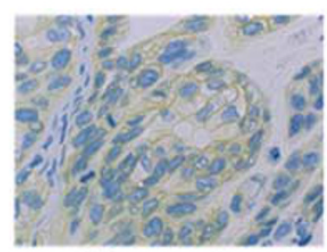

Coumarin

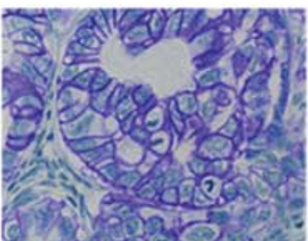

QSY09

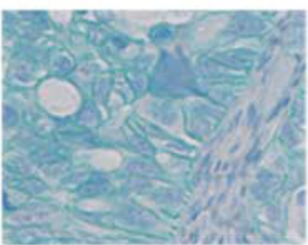

Malachite Green

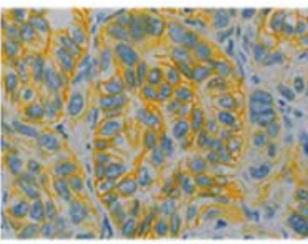

DABSYL

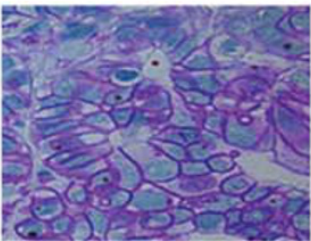

Sulforhodamine B

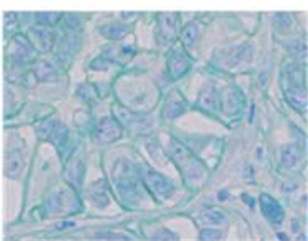

Erioglaucine

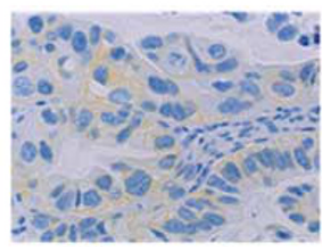

Fluorescein

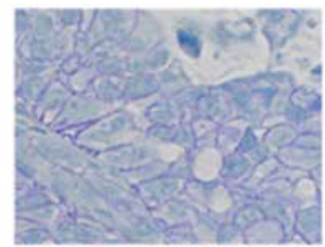

Sulforhodamine 101

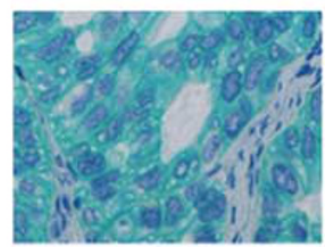

Cy5

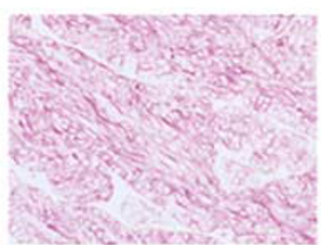

Rhodamine 110

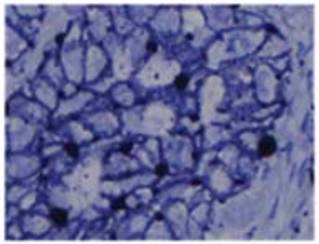

Gallocyanine

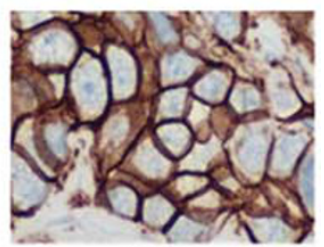

DAB

b

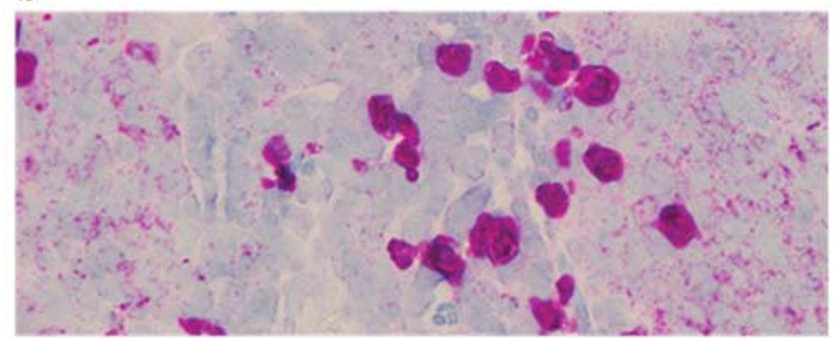

Tetramethylrhodamine

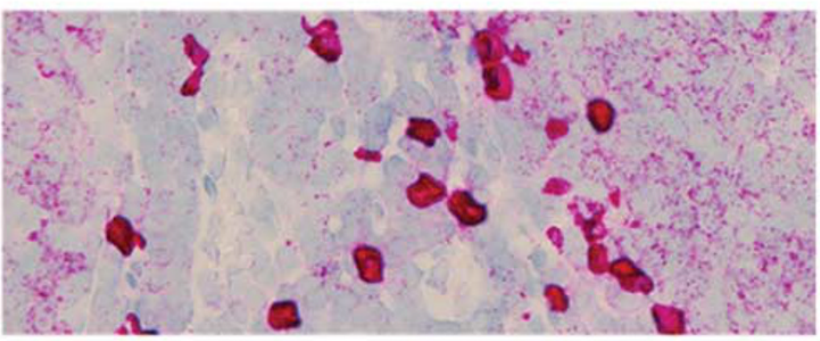

Sulforhodamine B

Figure 2 (a) Immunohistochemical (IHC) membrane Her2 protein staining of FFPE Calu-3 xenograft tissue in situ using tyramine-dye chromogens and conventional DAB chromogen. (b) In situ hybridization staining of KAPPA constant light chain mRNA in reactive tonsil.

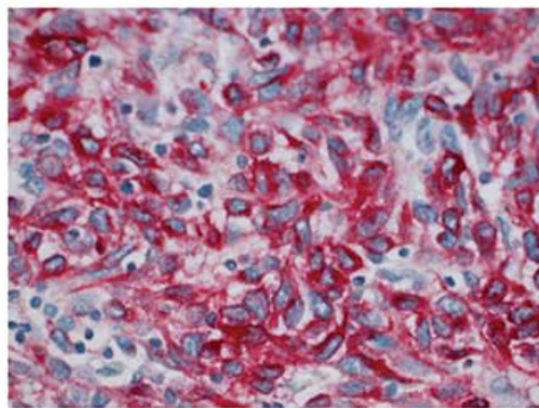

Fast Red

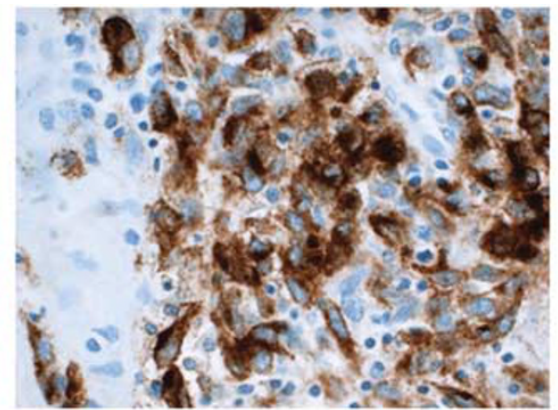

DAB

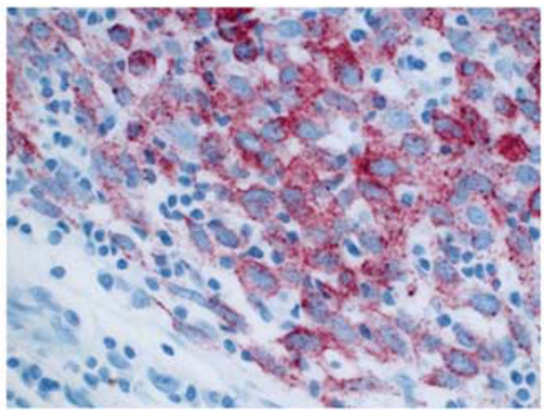

Tyramine-TAMRA

Figure 3 HMB45 protein containing granuole IHC staining in FFPE melanoma melanocytes using conventional chromogens (Fast Red, DAB) and tyramide-chromogen. 

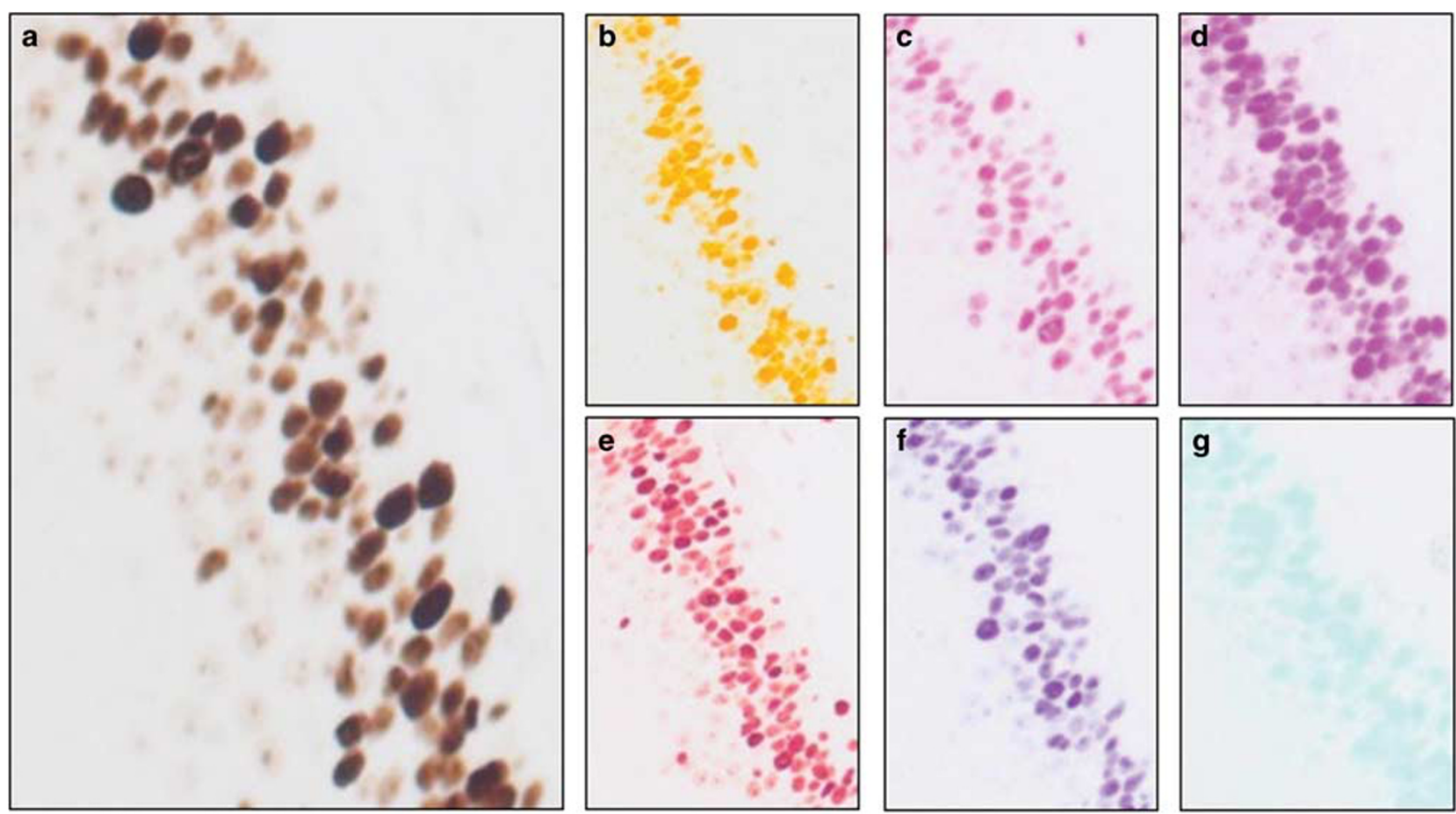

g
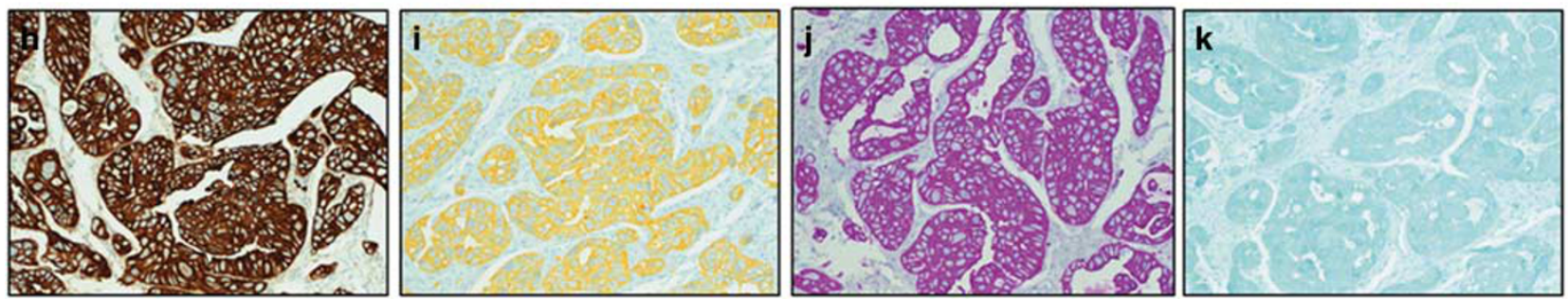

Figure 4 Detection in situ of nuclear and cytoplasmic proteins expressed over a wide dynamic range using tyramide-dye chromgens. (a-g) Nuclear Ki-67 IHC protein staining in reactive tonsil. (a) Diaminobenzidine (DAB) chromogen; (b) tyramide-dabsyl; (c) tyramide-rhodamine 6G; (d) tyramideTAMRA; (e) tyramide-rhodamine 110; (f) tyramide-sulforhodamine 101; (g) tyramide-Cy5. (h-k) Cytoplasmic cytokeratin IHC protein staining in reactive tonsil. (h) DAB chromogen; (i) tyramide-dabsyl; (j) tyramide-TAMRA; (k) tyramide-Cy5.

multiple biomarkers by providing an easy route to developing chromogens with narrow and selectable absorbance spectra. Accordingly, specific dyes providing minimal spectral overlap were selected from the new chromogen library and their utility determined for detection of multiple proteins differentially expressed on lymphocyte membranes. Figure 6a depicts staining of a breast tumor FFPE section for cytokeratin with TAMRA-tyramide (magenta), CD8 with a Cy5-tyramide/dabsyl-tyramide blend (green), and CD68 with rhodamine 110-tyramide (reddish-orange). Figure 6b depicts staining of a reactive tonsil FFPE section for CD8 with TAMRA-tyramide (magenta), Ki-67 with a Cy5-tyramide/ dabsyl-tyramide blend (green), and CD3 with dabsyltyramide (yellow). Used in a multiplex format the chromogens generated signals consistent with those observed with DAB (not shown). Moreover, each biomarker and cognate signal was distinguishable from other biomarker-chromogen signals and enabled analysis of each biomarker's expression in the model tissue.

\section{DISCUSSION}

Conventional chromogens, widely used for decades in immunohistochemical assays, provide sensitive signal discrimination over a useful dynamic range. However, the broad spectral properties and dependence on precipitation that established these gold standard chromogens limit their utility for multiplexed detection and analysis of multiple biomarkers. Owing to the entrenched nature of conventional chromogens and their familiarity to clinicians, new chromogens would ideally generate signals concordant with established chromogens (DAB), although having spectral properties that enable generation of unique signals visually discernable to clinicians and unmixable using imaging and computer algorithms. In addition, routine use of new chromogens should be consistent with current tissue slide workflows including dehydration and slide mounting.

Development of new chromogens with both the desired spectral properties and the solubility changes necessary for conventional precipitation-dependent deposition is a difficult 
a

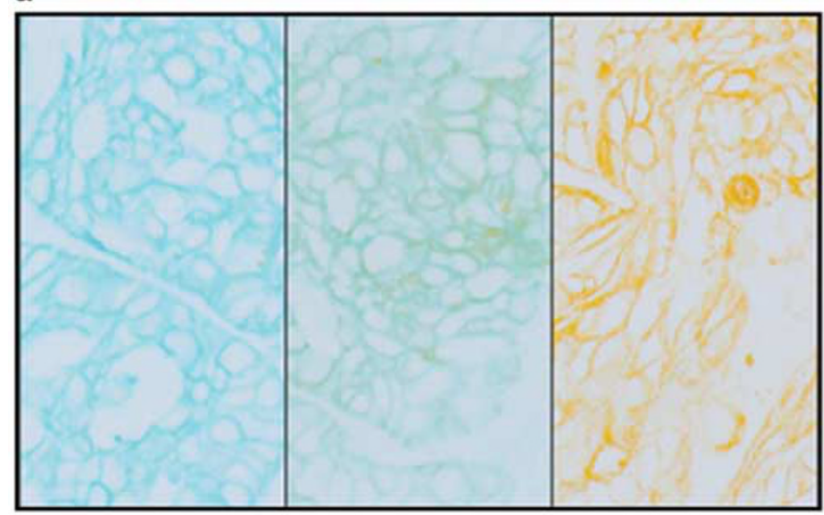

C

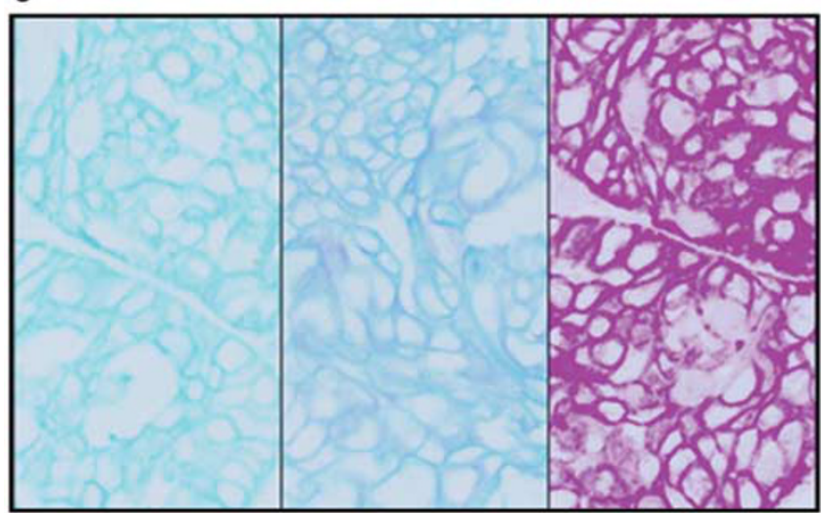

b

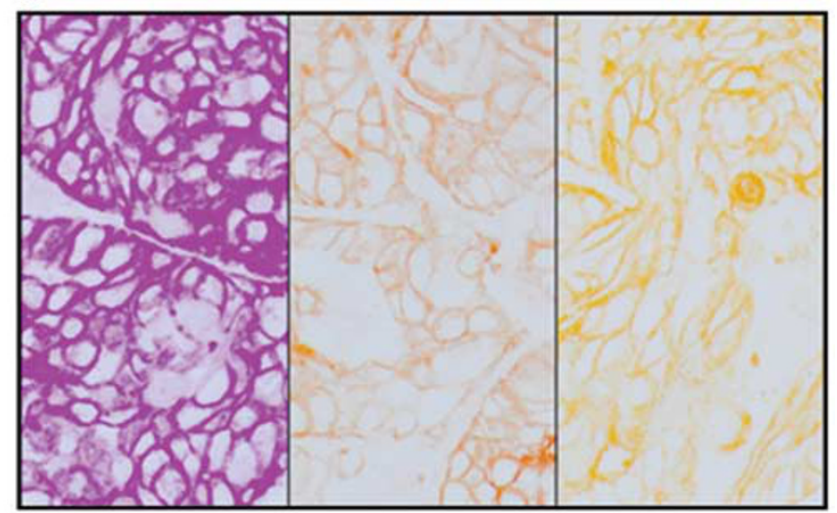

d

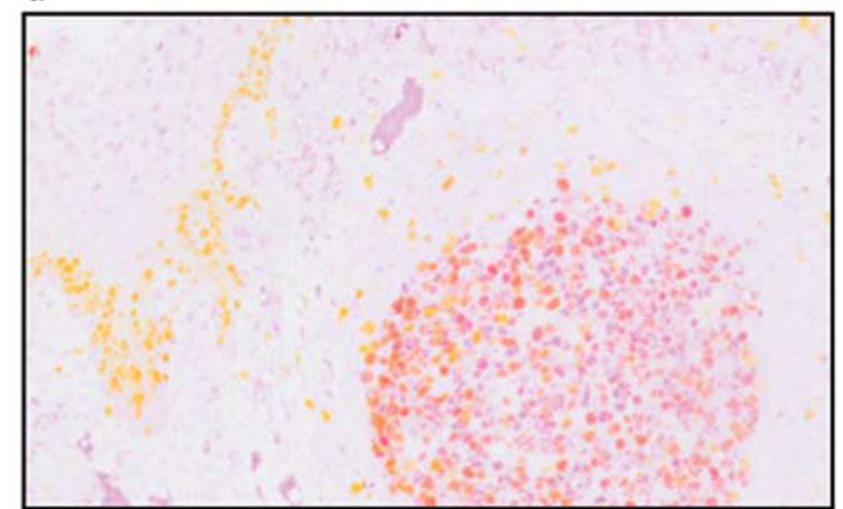

Figure 5 Generation of novel colors using blends of tyramide-dye chromogens and co-localization of two distinct protein biomarkers. (a) Green blend, left image, tyramide-Cy5; center image, tyramide-Cy5/dabsyl blend; right image, tyramide-dabsyl. (b) red blend; left image, tyramide-TAMRA; center image, tyramide-TAMRA/dabsyl blend; right image, tyramide-dabysl. (c) blue blend; left image, tyramide-Cy5; center image, tyramide-Cy5/TAMRA blend; right image, tyramide-TAMRA. (d) Co-localized protein biomarker color blend; nuclear Bcl-6 IHC protein stained using tyramide-TAMRA, nuclear Ki-67 IHC protein stained using tyramide-dabsyl.
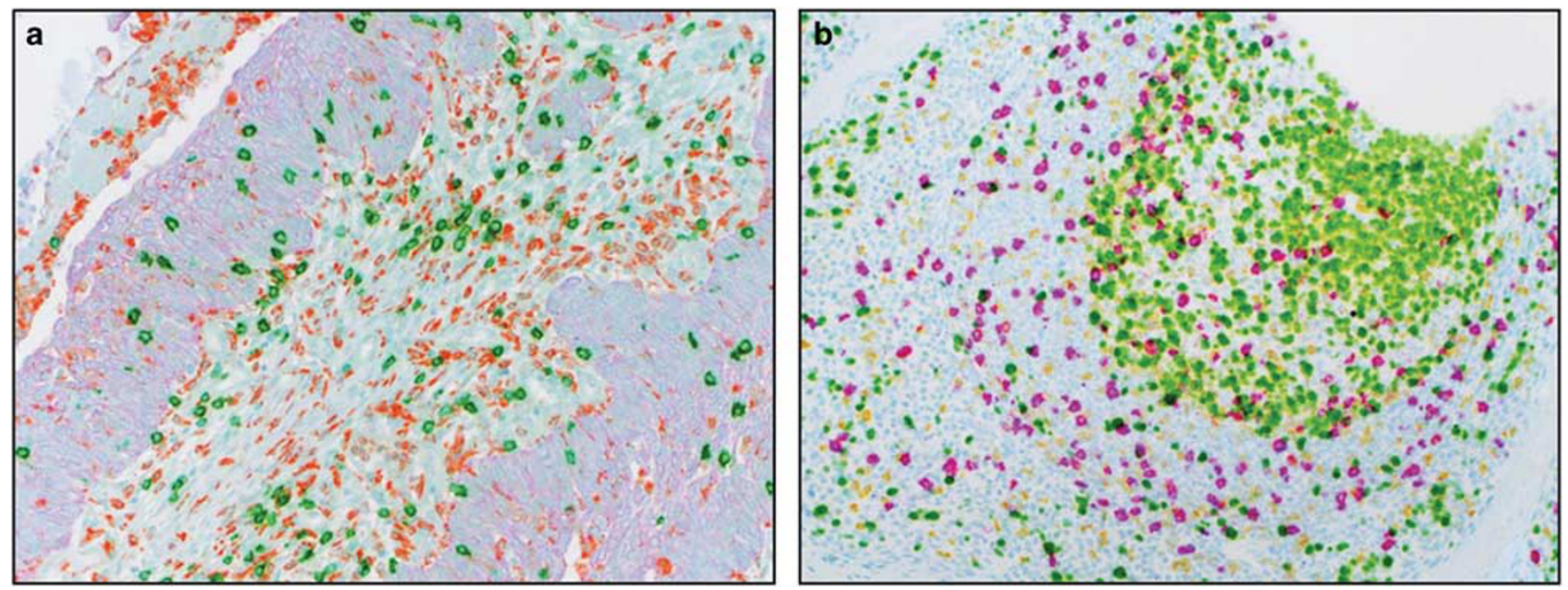

Figure 6 Multiplexed protein detection using tyramide-dye chromogens. (a) Three protein biomarker detection in breast cancer clinical tissue: cytoplasmic cytokeratin stained with tyramide-TAMRA (purple), membrane CD8 stained with tyramide-cy5/dabsyl blend (green), and membrane CD68 stained with tyramide-rhodamine 110 (orange). (b) Three protein biomarker detection in reactive tonsil tissue: membrane CD8 stained with tyramideTAMRA (purple), nuclear Ki-67 stained with tyramide-Cy5/dabsyl blend (green), and membrane CD3 stained with tyramide-dabsyl (yellow). 
process. To circumvent the solubility issues, the new chromogens described here rely on covalent deposition, and are generated by conjugating dyes with the desired spectral properties to tyramine molecules, as has been reported previously for fluorescent dyes, but not reported previously to covalently deposit dyes for visualization in brightfield microscopy. A large number of well-characterized dyes are available commercially, and we successfully conjugated 14 dyes to form dye-tyramides that generated visible chromogenic signals by IHC, amounting to an $82 \%$ success rate for all conjugations attempted, and demonstrating the ease of generating new chromogens by this approach. Table 1 lists seven of the dye-chromogens that span the visible spectrum and were further optimized for multiplexed IHC. Comparisons of the peak wavelengths of absorbance and widths of these peaks (FWHM) for the unconjugated, conjugated, and covalently deposited dyes show that most candidate dyes can be selected based on their spectral properties and visual color without having to first conjugate the dyes and use them in IHC, thereby accelerating the development process. Staining that matched DAB was achieved across many tissue types and dynamically expressed biomarkers; and data are shown in Figure 3 suggesting a staining pattern more consistent with specific biomarker biology. Since covalent deposition uses the same peroxidase-antibody reagents used in conventional chromogenic IHC, it fits well into current lab workflows.

Coloration observed in brightfield microscopy is governed by subtractive color mixing 22 and herein lays the value of chromogens with narrow absorbance bands. As light passes through the specimen the various colored components within the specimen absorb portions of the light depending upon their concentrations at a particular location and their absorbance spectra. Each colored component, therefore, subtracts light, reducing the intensity and changing the spectral distribution of the light transmitted to the eye or camera. Broadly absorbing chromogens remove a larger portion of the light spectrum, as seen in the absorbance spectra of the conventional DAB, Fast Red, and Fast Blue chromogens (Figure 1b), and co-localization of any two of these dyes removes essentially the entire visible spectrum such that Fast Red co-deposited with Fast Blue should be very difficult to distinguish from co-deposited DAB and Fast Red or co-deposited DAB and Fast Blue. This is consistent with the rules of color combination in the subtractive color system in which red and blue are secondary colors and their combination is black (no light transmitted). The primary colors in the subtractive system are yellow, magenta, and cyan, which are generated by dyes possessing relatively narrow single absorbance bands, such as the new chromogens listed in Table 1 (eg, dabsyl-yellow, TAMRA—magenta, and Cy5-cyan). In the subtractive system mixing yellow and magenta forms red, yellow and cyan forms green, and magenta and cyan form blue as demonstrated in Figure $5 \mathrm{a}$. Blending of the new chromogens, therefore, can be used to produce new stain colors. However, perhaps more importantly, we demonstrate that co-localization of two biomarkers, each one stained via a different cognate antibody with a different associated chromogenic stain, can produce a third distinctly colored stain (Figure 5d).

In addition to providing distinct coloration of co-localized dyes, dyes with narrow absorbance spectra each absorb a smaller part of the visible spectrum such that more dyes with distinct coloration can be used simultaneously. This is demonstrated in Figure 6 showing two examples of three biomarkers detected simultaneously, with two biomarkers in each example stained by individual chromogens and the third by a chromogen blend. Moreover, the ability to differentiate individual biomarker signals by means of digital imaging and spectral unmixing should be enhanced by the use of dyes with narrow absorbance bands.

Given the high success rate of converting candidate dyes to tyramide chromogens that enable visible in situ staining, it is puzzling that dye-tyramides have not been used as chromogens previously. One reason may be that tyramide amplification is often associated with high background staining. Generation of a visible chromogenic signal typically requires the deposition of large amounts of dye, 100 to 1000 times more dye than required to generate a visible fluorescent signal. Driving the enzymatic reaction to such high levels of deposition might be expected to exacerbate the background staining problem. The literature also suggests that high concentrations of dye-tyramide substrates necessary for increased deposition would result in tyramide dimerization in solution, rather than attachment to tissue. ${ }^{11}$ A recent report warns that peroxidase catalyzed tyramide reactions must be performed at low tyramide conjugate concentrations $(80 \mu \mathrm{M})$ to avoid dimerization. ${ }^{23}$ Last, even if tyramide amplification could be driven to higher levels, the tissue might not contain a sufficiently large number of attachment sites.

In work reported here, we were not limited by use of high dye-tyramide concentrations, and were able to covalently deposit dyes at substrate concentrations greater than $1 \mathrm{mM}$. Heavy deposits of dye necessary for visualization in brightfield microscopy were easily achieved for many dyes (Figure 1) and high background staining was avoided. By absorbance measurements of RNA ISH staining, 6-30 million molecules of chromogen were found to be deposited per $\mu \mathrm{m}^{2}$ in lightly to darkly stained punctate spots indicating that availability of binding sites is not limiting in tissue.

In summary, we have generated a new class of chromogens that stain tissue for specific biomarkers by covalent deposition of selected dyes conjugated with tyramine. This provides a simple and rapid route to developing new chromogens and permits selection of dyes with narrow absorbance bands that facilitates multiplexing, enabling simultaneous use of more dyes, permitting creation of new stain colors by blending chromogens, and allowing co-localized biomarkers to be easily distinguished from individual biomarkers. The new chromogens were remarkably concordant, in both signal morphology and dynamic range, with the broad absorbing 
DAB chromogen and are compatible with the workflow of conventional IHC.

Supplementary Information accompanies the paper on the Laboratory Investigation website (http://www.laboratoryinvestigation.org)

\section{DISCLOSURE/CONFLICT OF INTEREST}

All authors are employees of Ventana Medical Systems, (Ventana) a member of the Roche Group, in Tucson, AZ, USA. Ventana is the tissue diagnostic division of Roche Diagnostics and is a manufacturer of automated immunohistochemical and in situ hybridization assays.

1. Brankley SM, Wang KK, Harwood AR, et al. The development of a fluorescence in situ hybridization assay for the detection of dysplasia and adenocarcinoma in Barrett's esophagus. J Mol Diagn 2006;8:260-267.

2. Gerami $\mathrm{P}$, Jewell SS, Morrison LE, et al. Fluorescence in situ hybridization (FISH) as an ancillary diagnostic tool in the diagnosis of melanoma. Am J Surg Pathol 2009;33:1146-1156.

3. Sokolova IA, Bubendorf $L$, O'Hare $A$, et al. A fluorescence in situ hybridization-based assay for improved detection of lung cancer cells in bronchial washing specimens. Cancer 2002;96:306-315.

4. Nakane PK, Pierce GB. Enzyme-labeled antibodies: Preparation and application for the localization of antigens. Journal of Histochemistry and Cytochemistry 1966;14:929-931.

5. Mason DY, Sammons R. Alkaline phosphatase and peroxidase for double immunoenzymatic labelling of cellular constituents. J Clin Pathol 1978;31:454-460.

6. van der Loos CM. Chromogens in multiple immunohistochemical staining used for visual assessment and spectral imaging: the colorful future. J Histotechnol 2010;33:31-40.

7. Petersen KH. Novel horseradish peroxidase substrates for use in immunohistochemistry. J Immunol Methods 2009;340:86-89.

8. Adler K, Erickson T, Bobrow M. High sensitivity detection of HPV-16 in $\mathrm{SiHa}$ and CaSki cells utilizing FISH enhanced by TSA. Histochem Cell Biol 1997;108:321-324.

9. Bobrow MN, Moen Jr PT. Tyramide signal amplification (TSA) systems for the enhancement of ISH signals in cytogenetics. Curr Protoc Cytom 2001:Chapter 8:Unit 89.

10. van Gijlswijk RP, Zijlmans HJ, Wiegant J, et al. Fluorochrome-labeled tyramides: use in immunocytochemistry and fluorescence in situ hybridization. J Histochem Cytochem 1997;45:375-382.

11. Bobrow MN, Harris TD, Shaughnessy KJ, Litt GJ. Catalyzed reporter deposition, a novel method of signal amplification. Application to immunoassays. J Immunol Methods 1989;125:279-285.
12. Bobrow MN, Shaughnessy KJ, Litt GJ. Catalyzed reporter deposition, a novel method of signal amplification. II. Application to membrane immunoassays. J Immunol Methods 1991;137:103-112.

13. Adams JC. Biotin Amplification of Biotin and Horseradish-Peroxidase Signals in Histochemical Stains. J Histochem Cytochem 1992;40: 1457-1463.

14. Raap AK, van de Corput MP, Vervenne RA, et al. Ultra-sensitive FISH using peroxidase-mediated deposition of biotin- or fluorochrome tyramides. Hum Mol Genet 1995;4:529-534.

15. Kerstens HMJ, Poddighe PJ, Hanselaar AGJM. A novel in situ hybridization signal amplification method based on the deposition of biotinylated tyramine. J Histochem Cytochem 1995;43: 347-352.

16. Chao J, DeBiasio R, Zhu Z, et al. Immunofluorescence signal amplification by the enzyme-catalyzed deposition of a fluorescent reporter substrate (CARD). Cytometry 1996;23:48-53.

17. Hopman AH, Ramaekers FC, Speel EJ. Rapid synthesis of biotindigoxigenin-, trinitrophenyl-, and fluorochrome-labeled tyramides and their application for In situ hybridization using CARD amplification. J Histochem Cytochem 1998:46:771-777.

18. Stack EC, Wang C, Roman KA, Hoyt CC. Multiplexed immunohistochemistry, imaging, and quantitation: a review, with an assessment of Tyramide signal amplification, multispectral imaging and multiplex analysis. Methods 2014;70:46-58.

19. Speel EJ, Ramaekers FC, Hopman AH. Sensitive multicolor fluorescence in situ hybridization using catalyzed reporter deposition (CARD) amplification. J Histochem Cytochem 1997:45:1439-1446.

20. Raj A, Peskin CS, Tranchina $D$, et al. Stochastic mRNA synthesis in mammalian cells. PLoS Biol 2006;4:e309.

21. Rimsza LM, Day WA, McGinn S, et al. Kappa and lambda light chain mRNA in situ hybridization compared to flow cytometry and immunohistochemistry in B cell lymphomas. Diagn Pathol 2014;9: 144.

22. Berns RS. Principles of Color Mixing third ed. John Wiley \& Sons: New York, 2000.

23. Lohse J, Petersen $\mathrm{KH}$, Woller NC, et al. Improved catalyzed reporter deposition, iCARD. Bioconjug Chem 2014;25:1036-1042.

24. Lin J-K, Chang J-Y. Chromophoric labeling of amino acids with 4-dimethylaminoazobenzene-4'-sulfonyl chloride. Analyt Chem 1975:47(9):1634-1638.

25. Hameau A, Fuchs S, Laurent $R$, et al. Synthesis of dye/fluorescent functionalized dendrons based on cyclotriphosphazene. Beilstein J Org Chem 2011;7:1577-1583.

26. Chadwick CS, McEntergart MG, Nairn RC. Fluorescent protein tracers; a trial of new fluorochromes and the development of an alternative to fluorescein. Immunology 1958;1:315-327. 